\title{
Insight and major mental illness: an update for clinicians
}

\section{Simon Surguladze \& Anthony David}

This article will concentrate on insight into serious mental disorder. Not psychoanalytic insight - nor indeed, the insight all of us have, to a greater or lesser extent, into our own attitudes, motives and behaviour - but the insight which patients with psychosis have into their mental pathology. This is no longer considered an all-or-none phenomenon, but rather a dimensional one, so that subjects can have different levels of awareness into their illness. The suggestion was first mooted by Aubrey Lewis (1934) in his seminal work on insight. Conceptual exploration of insight has been activated in the past decade and is proceeding in parallel with the construction of special scales to measure insight and research into its cognitive, biological, social and cultural basis.

\section{Concepts and scales}

It is generally accepted that insight is, at its simplest, a dimension, and probably a complex series of partially overlapping dimensions. Rather than committing to a single definition, researchers and clinicians have devised scales which they hope capture the essence of the construct. One approach (David, 1990) has been to distinguish three main aspects which are listed in Box 1.

Some workers in the field have expanded upon these dimensions, proposing a separation of the processes underlying awareness and those of attribution (Amador \& Strauss, 1990). Kemp \& David (1997) suggested that awareness of change was also a prerequisite of insight which could fall short of a medical attribution of illness. Furthermore, there has been much interest in the specificity of insight for certain specific features of mental disorder such as positive $v$. negative symptoms and mood disorder $v$. abnormal perceptions. There are now a number of psychometric instruments and scales to assess insight which are set out in Box 2.

It should be emphasised, however, that there is correspondence between most scales in covering such aspects as acceptance of the illness 'label', and perceived need for treatment (for a review see Amador \& Seckinger, 1997; Sanz et al, 1998).

Depending on their particular aims, clinicians or researchers can now choose the appropriate tool from the instruments available. A single-item insight assessment of the traditional mental state examination provides the investigator with little information about the quality or quantity of insight which

Box 1. Multi-dimensional model of insight

Awareness that one is suffering, in a general way, from a mental (as opposed to a physical) disturbance which could be an illness

More specific awareness that certain experiences including beliefs and perceptions may not be veridical, and further that they too could be part of an illness

Acknowledgement of the medical implications of the above, a concrete token of which is informed acceptance of treatment

Simon Surguladze, Doctor of Science in psychiatry, is a Senior Research Worker at the Institute of Psychiatry, Tbilisi, Georgia (former Soviet Union). His recent work has focused on social attitudes and insight in patients with schizophrenia. He is currently conducting a study on cognitive disturbances in schizophrenia at the London Institute of Psychiatry and is supported by a Wellcome Trust Travelling Fellowship. Anthony David is Professor of Cognitive Neuropsychiatry at the Institute of Psychiatry (De Crespigny Park, London SE5 8AF) and GKT School of Medicine, and honorary consultant psychiatrist at the Maudsley Hospital. His research interests include insight in psychoses, neuropsychology of schizophrenia and neuroimaging. 
Box 2. Instruments assessing insight

Insight and Treatment Attitude Questionnaire - ITAQ (McEvoy et al, 1989a): an 11 dichotomous item, clinician-administered questionnaire weighted towards treatment compliance

Scale to Assess Unawareness of Mental Disorder - SUMD (Amador \& Strauss, 1990): a structured interview which divides current and past disorder, awareness and attribution, benefit from treatment, and the psychosocial consequences of having a mental disorder

Schedule for the Assessment of Insight - SAI (David, 1990): a simple semi-structured clinician-administered series of probes on the three dimensions (rated on three levels) and the expanded version - SAI-E (Kemp \& David, 1997) - which includes awareness of change and its effects on the person's functioning

Insight Scales A \& B (Markova \& Berrios, 1992): self- or observer-administered scales. Apart from psychopathologyrelated issues, the instrument explores self-knowledge relevant to social and environmental situations

Insight Scale - IS (Birchwood et al, 1994): a self-report scale based around similar dimensions to the SAI

Awareness of Illness Interview - AII (Cuffel et $a l, 1996)$ : a semi-structured interview covering two broad factors - recognition of illness and the perceived need for treatment

There are also several items touching on insight in standard psychopathological assessment scales - for example, in the Positive and Negative Syndrome Scale for Schizophrenia (Kay et $a l, 1987$ ) and the Present State Examination (Wing et al, 1974)

may be of interest in epidemiological studies, or in everyday psychiatric practice. The appearance of 'poor insight', 'good insight' or even 'partial insight' in psychiatric case notes should be a thing of the past. Comment needs to made as to which aspects of the whole presentation of the therapist's formulation the patient accepts. Does this vary from moment to moment or as the illness evolves? How fixed is it? Does illness awareness lead inevitably to treatment adherence, or is there no connection? Do the patient's actions indicate some awareness of their illness as opposed to their overt statements? Detailed longitudinal monitoring in clinical settings and psychopathology research requires more structured measurement of insight - which is the feature of the scales described above.

\section{Insight and psychopathology}

\section{Schizophrenia}

Traditionally, psychotic symptoms and lack of insight were considered to be two sides of the same coin, or the phenomena were supposed to underlie each other (a rather circular relationship). Furthermore, schizophrenia was held up as the epitome of a disorder with lack of insight. Indeed, impairment of insight was the most common and discriminating symptom for schizophrenia in the World Health Organization's (1973) International Pilot Study of Schizophrenia. More recent work using more complex (non-tautological) definitions of insight (Amador et al, 1994) found that lack of insight was common in schizophrenia (and psychotic bipolar disorder), but less so in other affective and schizoaffective disorders diagnosed as part of the DSM-IV field trials.

There are a number of recent studies exploring the association of insight and psychopathological symptoms in patients with schizophrenia. McEvoy et al (1989b) found a moderately significant association between insight and Brief Psychiatric Rating Scale scores (BPRS; Overall \& Gorham, 1962), which became less significant by the time of discharge. Thus, as the psychopathology of the schizophrenic episode showed changes toward improvement, lack of insight remained a more stable variable. David $e t$ al (1992) revealed a moderate though significant $(-0.31)$ correlation between Present State Examination total scores and a composite score in the Schedule for Assessment of Insight (SAI). This study did not find significant differences in insight scores between the patients with schizophrenia and the rest, comprising patients with paranoid psychoses and affective psychoses. Surprisingly, patients with hallucinations did not differ from others in their level of insight. Several other investigators (Michalakeas et al, 1994; Amador et al, 1994) have found significant correlations between insight and psychopathological state, although the range of variance, explained by this correlation was generally low less than $50 \%$.

Jorgensen (1995) questioned the directness of the relationship between symptoms and insight by studying recovery from a psychotic (schizophrenic) 
episode. Considering different patterns of 'delusional dynamics' as patients approached remission, the author found that level of insight increased independently of the type of recovery. Subsequently, insight was suggested to represent a distinct component of recovery, separate from (other) positive symptoms. One could conjecture, therefore, that despite some overlap, the mechanisms underlying psychotic symptoms and those of (lack of) insight may not be identical. It has been suggested (Kim $e t$ $a l, 1997 a, b)$ that insight might be more strongly related to the subjective experiences of patients, as opposed to observer-rated 'behavioural' measures. Kim and co-workers constructed an interview exploring subjective components of psychotic symptoms from standardised rating scales. They revealed a moderate inverse correlation between observer-rated positive symptoms and insight measures (as assessed by the BPRS and David's SAI scale, respectively). However, the subjective experiences of psychosis did not correlate with the measure of insight, contrary to the authors' hypothesis. It is, of course, possible that observer ratings of psychopathology contaminated observer ratings of insight.

\section{Insight in mood disorders}

Assessment of insight could shed some light on clinical evaluation of affective/post-affective states. Patients with bipolar disorders, investigated by the Insight and Treatment Attitude Questionnaire (McEvoy et al, 1989a) showed that insight was severely impaired in mania (comparable to that in schizophrenia), and less impaired in depressive states (Michalakeas et_al, 1994; Ghaemi et al, 1995). These studies also reported different results regarding changes in insight over time compared to schizophrenia: the first study found improvement of insight after recovery from a manic state, and the second, that there was no improvement. Similarly, Peralta \& Cuesta (1998) showed that, on admission, patients with mania had greater impairment of insight than those with depression, and at discharge, some impairment was still observed in the patients with mania, particularly those with moodincongruent psychotic symptoms. The relationship between 'recovery' and insight is, again, prone to circularity. Amador \& Seckinger (1997) argued on pragmatic grounds that there is no need for the patient overtly to accept a diagnosis of mental disorder, so long as they are able to benefit from treatment.

Focusing on depressive disorders, Ghaemi et al $(1995,1997)$ found that patients with seasonal affective disorder possessed a moderate amount of insight (as measured by the Scale to Assess Unawareness of Mental Disorder; Amador \& Strauss, 1990) into depressive symptoms, which did not change after recovery. Increased severity of illness in seasonal affective disorder was associated with increased insight into depressive symptoms. A positive correlation between insight and depressive states was also found by Sanz et al (1998). These findings are consistent with the concept of depressive realism (Alloy \& Abramson, 1988), which implies that in some cases, depression may result from excessive insight into unfavourable life circumstances.

It is worth mentioning here that lack of insight is not solely concerned with psychotic or severe affective states - insight may sometimes be impaired in 'neurotic' diseases, such as obsessive-compulsive disorder (Eisen et al, 1994) and anorexia nervosa (Feighner et al, 1972). These are, however, outside the scope of the present article.

\section{Lay models of illness}

Can unawareness of illness be caused merely by lack of knowledge? (That is, patients cannot re-label their experiences as pathological, because they do not know the criteria of mental disorder.) However plausible this explanation may seem, supporting evidence is lacking. Startup (1997) discovered a close to normal ability of patients to recognise the symptoms of mental illness in others (as tested using case vignettes), but this was in stark contrast to their impaired awareness of illness in themselves (as inferred from scores on the Insight and Treatment Attitude Questionnaire). McEvoy et al (1993) had earlier shown that patients with schizophrenia admitted more readily to (or were more aware of) negative symptoms than positive ones (see also Amador et al, 1994). At the same time, patients considered negative features as least typical of mental illness (cf. positive symptoms). Moreover, the study revealed selectivity of (un)awareness towards those negative symptoms which could be construed as most pejorative (e.g. loss of motivation). This is in line with work by Swanson et al (1995), where patients with schizophrenia and mania were shown vignettes describing examples of positive, negative and manic psychopathology. On admission, patients with schizophrenia rated themselves as significantly less similar to the positive symptom vignettes than psychiatrists rated them, although the patients correctly labelled the presented signs as pathological. Ratings from patients with mania did not differ from the psychiatrists' rating of their 
similarity to the vignettes, but patients strongly denied that the vignettes reflected mental illness. It is likely that at least in some cases, apparent lack of insight might be determined by the reluctance of the patients to admit having socially undesirable characteristics.

\section{Insight and neuropsychological performance}

Studies of the neuropsychological substrate of insight impairment in schizophrenia call to mind an earlier search for the "primary symptoms... stemming from the disease process itself" (Bleuler, 1911, p. 348). The debates around the issue of a neuropsychological substrate of insight fall into two camps: one which maintains that there are specific neuroanatomical sites (e.g. frontal lobes) which are responsible for the impairment of insight in schizophrenia, and the other which claims that, if insight is related to cognitive impairment, it results from a global cognitive deficit.

Two research groups (Young et al, 1998; Lysaker et al, 1998a) have shown and replicated a correlation between poor insight (measured by the Scale to Assess Unawareness of Mental Disorder) and executive deficits, measured by the Wisconsin Card Sorting Test (WCST). Other groups including Cuesta et al (1995), Collins et al (1997) and Sanz et al (1998) failed to find a significant relationship between insight and frontal task (WCST) performance. David et al (1995) suggested that insight is related not specifically to frontal, but more to measures of general intelligence, although these relationships were found to be non-linear.

The WCST is problematic as a measure of executive or 'frontal' dysfunction, being dependent on general cognitive abilities (see Dieci et al, 1997; Laws, 1999). Furthermore, Rossell \& David (1997) were able to demonstrate that impaired performance of patients with schizophrenia in the WCST is not fixed but can be improved using the verbalisation of responses which may enhance error detection.

More subtle approaches to the question of insight and cognitive impairment have been taken. Cuesta et al (1996) investigated the relationships between subjective experiences in schizophrenia (as measured by the Frankfurt Complaints Questionnaire (Sullwold, 1986)) and neuropsychological performance. As in the study by Kim et al (1997b), subjective experiences did not correlate with insight, but scores were highly correlated with neuropsychological performance. This rules out a global deficit in detection of impairment. Of much interest is the study of Startup (1996), who found that the relationships between neuropsychological performance and insight in schizophrenia are of a complex curvilinear nature. He suggested that, besides cognitive deficits, motivational forces could be implicated in the development of insight impairment (particularly in those of above average intelligence).

Our own view is that intellectual abilities whether specific frontal lobe functions, or more general abilities - may be meaningfully associated not just with general scores of insight, but with more specific tasks requiring cognitively-demanding introspection into particular domains of mental life.

\section{Cultural influences on insight}

There are at least two simple ways in which external influences may shape insight measures. First, as hinted at above in the discussion of 'motivational accounts' of illness unawareness, patients may consciously or unconsciously avoid admitting that they are suffering from pathological symptoms because of their unwillingness to bear the stigma of mental illness. This has parallels with the intellectual $v$. emotional insight distinction of psychoanalysis. However, the patient may differ from the investigator in terms of educational/cultural background, as well as on a theoretical continuum of conformity, and subsequently in their explanations of the experience of mental disorder. The vignette studies do not support this notion, but it cannot yet be dismissed. In this respect, several investigators (Perkins \& Moodley, 1993; Johnson \& Orrell, 1995, 1996) have argued that patients may have their own explanations of their disturbances (e.g. religious, physical, interpersonal) which may not coincide with the Western 'medical model' of mental disease. The situation can be even more complicated if one tries directly to impose the models of insight on patients from non-Western cultures. Referring to his experience in Singapore, Tan (1993) warns that allowances should be made when assessing components of insight. For example, treatment compliance might be applied to either traditional remedies or 'modern' treatments. The extent to which the ability to distance oneself from unusual experiences is dependent on cultural norms is a topic for future multi-disciplinary research (see Box 3).

Beck-Sander (1998) criticises the concept of insight as being restrictive and internally inconsistent (but see David, 1998). As an alternative, an individual explanatory model is proposed, which is grounded on the patient's explanation of their illness. Nevertheless, there are benefits to the patient in 
embracing the 'medical model', provided it does not imply incurability and lack of autonomy. In many cases individual explanations and insight are not mutually exclusive but complementary. Medical professionals should, however, be aware of patients' use of terms referring to their mental state. McNally \& Goldberg (1997) avoided terms like 'illness' and 'symptom' when interviewing patients with schizophrenia about their subjective experiences and natural coping strategies. The patients, scoring medium to high on the positive symptoms scale of the Positive and Negative Syndrome Scale (Kay et al, 1987), reported such coping strategies as 'trying to stand outside of one's experience and see it from the perspective of another', 'reality checks', etc. Although these descriptions of self-developed strategies would not coincide literally with items on insight scales, they demonstrated an implicit awareness of having a mental disorder. Indeed, insight positively correlated with the number of natural coping strategies employed by patients with auditory hallucinations in one survey (Nayani \& David, 1996).

Regarding the influence of culture on coping mechanisms, Wahass \& Kent (1997) showed that patients with an Islamic background were more inclined towards religious activities to cope with their 'voices', while native British patients engaged in 'physiological' methods such as sport, using alcohol and taking medication. Considering these findings from an insight viewpoint, we should mention that not all of these coping strategies reflect presence of good insight. Thus, Sanz et al (1998) demonstrated, that the use of alcohol and illicit drugs correlated negatively with insight, which subsequently might hinder the adherence to medication. Again, activation of religious practices may be helpful for patients belonging to Islamic culture, but may be inappropriate in different settings. Kirov et al (1998) found in a recent study in

Box 3. Factors influencing insight in psychosis

Cultural models of illness and health

General intelligence and knowledge

Doctor-patient relationship

Symptomatology: lack of insight associated with delusions; preserved insight associated with depressed mood

Executive function deficits

'Denial' - motivation, preservation of selfesteem, avoidance of stigma

Personality - 'compliance non-conformity' as traits inner London, that religious affiliations in a group of patients recovering from psychotic relapse were not correlated with insight.

\section{Family attitudes}

The family is a potent transmitter of cultural and ancestral values. A few studies have begun to look at this more closely in relation to insight and attitudes to treatment. Smith et al (1997) attempted to study this by approaching the patient's nominated 'significant other' in order to examine his or her illness beliefs. The authors found that a third of these realtives were uncooperative. The patients shared similar attitudes, including failure to note the beneficial effects of medication. Families and carers generally may have experienced medication failures firsthand, or may have felt blamed by the medical profession for the patient's illness. They too may have experienced psychiatric disorders. Clearly, 'insight' cannot be located solely in the mind of the individual patient without acknowledging family circumstances.

\section{Coping with psychosis}

One very important area where research and practice are coinciding is in the early detection of, and intervention in, psychosis. The prodromal states of manic-depressive illness and schizophrenia are sometimes well-known to patients (or can be learned), thus enabling the implementation of strategies to prevent relapse (see Heinrichs et al, 1985).

In bipolar patients, Lam \& Wong (1997) showed different patterns of insight into the prodromal signs of mania $v$. depression $-25 \%$ of subjects reported that they could not detect any early warnings of depression, compared with only $7.5 \%$ of patients who could not detect prodromes of mania. The patients described various types of cognitive and behavioural strategies helping them to cope with detected symptoms. Insight (measured by the Schedule for the Assessment of Insight; David, 1990) was found to have an effect on coping with prodromes to mania only.

Huber \& Gross (1997) have introduced the concept of 'basic symptoms' of schizophrenia which represent subjectively apprehended minor deficits in cognitive, emotional and volitional functions (memory deficits, irritability, distractability, etc.). The authors argued that providing patients not only with pharmacotherapy, but also with skills in recognition of and coping with their basic symptoms, could be successful in primary prevention. The lack 
of a relationship between subjective awareness of symptoms and insight is, however, problematic for this view.

\section{Treatment}

The relevance of the concept of insight to therapeutic intervention is increasing. Given the changes in mental health care policy towards a communitybased system, the patient is regarded as the participant in (and no longer the mere recipient of) the treatment programme. The success of this approach depends to a large extent on the patient's insight and attitudes towards therapy. The association between insight and adherence to medication has been demonstrated in a number of studies (Chan, 1984; Buchanan, 1992; McEvoy, 1998). Insight has also been found to be associated with social functioning (Lysaker et al, 1998b).

Despite the most liberal attitudes, the sanctioning of involuntary treatment under the Mental Health Act is a fact of life for most British psychiatrists. Studies from the USA (McEvoy et al, 1989b) and the UK (David et al, 1992) confirm the rather obvious idea that poor insight is a particular feature of involuntary patients, and that it is quite stable in the face of clinical remission. Nevertheless, in some cases (over $50 \%$ of patients in the Kemp et al (1998) study), enforced compliance may be the first step towards voluntary treatment adherence (Kane et al, 1983; Kjellin et al, 1997).

Psychoeducation has been used to improve the knowledge base of patients and their families regarding the nature of mental illness and the benefits of medication. However, studies tend to show shortlived benefits at best (Boczkowski et al,1985; MacPherson et al,1996). An 18-month follow-up of a randomised controlled trial study by Kemp et al (1998) has shown that 'compliance therapy' demonstrated better outcome in terms of social functioning and community tenure compared with a control intervention. This therapy combines interactive, cognitive and behavioural approaches and promotes clinical improvement via attitudes towards treatment and the increase of insight into existing psychotic symptoms (see also Kemp et al, 1997).

\section{Conclusion}

There is growing evidence that insight into psychosis is a multi-dimensional process, unfolding with time. It is domain-specific and influenced by a host of factors (cognitive, emotional, social and cultural) which all interact. The concept of insight is far from being fully understood. There are many uncertainties regarding the association of insight with psychopathology and neuropsychological performance. Some of these uncertainties reflect the dynamic nature of insight, which implies a certain malleability and opens the door for therapeutic intervention.

In this article we have stressed the clinical implications of the insight concept, the meaningfulness of subjective attitudes of patients, and the fact that the quality of patients' lives might be improved by targeting insight and attitudes to treatment.

\section{References}

Alloy, L. B. \& Abramson, L. Y. (1988) Depressive realism: Four theoretical perspectives. In Cognitive Processes in Depression (ed. L. B. Alloy), pp.223-265. New York: Guilford Press.

Amador, X. F., Flaum, M., Andreasen, N. C., et al (1994) Awareness of illness in schizophrenia, schizoaffective and mood disorders. Archives of General Psychiatry, 51, 826-836.

- \& Strauss, D. H. (1990) The Scale to Assess Unawareness of Mental Disorder (SUMD). New York: Columbia University and New York State Psychiatric Institute.

— \& Seckinger, B. A. (1997) The assessment of insight: A methodological review. Psychiatric Annals, 27, 12, 798805.

Beck-Sander, A. (1998) Is insight into psychosis meaningful? Journal of Mental Health, 7, 25-34.

Birchwood, M., Smith, J., Drury, V., et al (1994) A self-report insight scale for psychosis - reliability, validity and sensitivity to change. Acta Psychiatrica Scandinavica, 89, 62-67.

Bleuler, E. (1911) (English edition 1950) Dementia Praecox, or The Group of Schizophrenias. New York: International Universities Press.

Boczkowski, J. A., Zeichner, A. \& De Santo, N. (1985) Neuroleptic compliance among chronic schizophrenic outpatients: an intervention outcome report. Journal of Consulting and Clinical Psychology, 53, 666-671.

Buchanan, A. (1992) A two-year prospective study of treatment compliance in patients with schizophrenia. Psychological Medicine, 22, 787-797.

Chan, D. W. (1984) Medication compliance in a Chinese psychiatric out-patient setting. British Journal of Medical Psychology, 57, 81-89.

Collins, A. A., Remington, G. J., Coulter, K., et al (1997) Insight, neurocognitive function and symptom clusters in chronic schizophrenia. Schizophrenia Research, 27, 37-44.

Cuesta, M. J., Peralta, V., Caro, F., et al (1995) Is poor insight in psychotic disorders associated with poor performance on the Wisconsin Card Sorting Test? American Journal of Psychiatry, 152, 1380-1382.

-, - \& Juan, J. A. (1996) Abnormal subjective experiences in schizophrenia: its relationships with neuropsychological disturbances and frontal signs. European Archives of Psychiatry and Clinical Neuroscience, 246, 101-105.

Cuffel, B. J., Alford, J., Fischer, E. P., et al (1996) Awareness of illness in schizophrenia and outpatient treatment adherence. Journal of Nervous and Mental Disease, 184, 653-659.

David, A. S. (1990) Insight and psychosis. British Journal of Psychiatry, 156, 798-809.

- (1998) The clinical importance of insight. In Insight and Psychosis (eds X. F. Amador \& A. S. David), pp.332-351. New York: Oxford University Press. 
- Buchanan, A., Reed, A., et al (1992) The assessment of insight in psychosis. British Journal of Psychiatry, 161, 599-602.

-, van Os, J., Harvey, I., et al (1995) Insight and psychotic illness. British Journal of Psychiatry, 167, 621-628.

Dieci, M., Vita, A., Silenzi, C., et al (1997) Non-selective impairment of Wisconsin Card Sorting Test performance in patients with schizophrenia. Schizophrenia Research, 25, 33-42.

Eisen, S. V., Dill, D. L. \& Grob, M. C. (1994) Reliability and validity of a brief patient-report instrument for psychiatric outcome evaluation. Hospital and Community Psychiatry, 45, 242-247.

Feighner, J. P., Robins, E., Guze, S. B., et al (1972) Diagnostic criteria for use in psychiatric research. Archives of General Psychiatry, 26, 57-63.

Ghaemi, S. N., Stoll, A. L. \& Pope, H. (1995) Lack of insight in bipolar disorder - the acute manic episode. Journal of Nervous and Mental Disease, 183, 464-467.

- Sachs, G. S., Baldassano, C. F., et al (1997) Insight in seasonal affective disorder. Comprehensive Psychiatry, 38, 345-348.

Heinrichs, D. W., Cohen, B. P. \& Carpenter, W. T. (1985) Early insight and the management of schizophrenia decompensation. Journal of Nervous and Mental Disease, 173, 133-138.

Huber, G. \& Gross, G. (1997) Advances in therapy and prevention of schizophrenic disorders. Neurology Psychiatry and Brain Research, 5, 1-8.

Johnson, S. \& Orrell, M. (1995) Insight and psychosis - a social perspective. Psychological Medicine, 25, 515-520.

- \& - (1996) Insight, psychosis and ethnicity - a casenote study. Psychological Medicine, 26, 5, 1081-1084.

Jorgensen, P. (1995) Recovery and insight in schizophrenia. Acta Psychiatrica Scandinavica, 92, 6, 436-440.

Kane, J. M., Quitkin, F., Rifkin, A., et al (1983) Attitudinal changes of involuntarily committed patients following treatment. Archives of General Psychiatry, 40, 374-377.

Kay, S., Fiszbein, A. \& Opler, L. (1987) The Positive and Negative Syndrome Scale (PANSS) for schizophrenia. Schizophrenia Bulletin, 13, 261-276.

Kemp, R. \& David, A. (1997) Insight and compliance. In Treatment Compliance and the Treatment Alliance in Serious Mental Illness (ed. B. Blackwell), pp. 61-86. The Netherlands: Harwood Academic.

-, Hayward, P., \& David, A. (1997) Compliance Therapy Manual. London: The Maudsley.

-, Kirov, G., Everitt, B., et al (1998) Randomised controlled trial of compliance therapy - 18-month follow-up. British Journal of Psychiatry, 172, 413-419.

Kim, Y., Sakamoto, K., Kamo, T., et al (1997a) Insight and clinical correlates in schizophrenia. Comprehensive Psychiatry, 38, 117-123.

-, - - Sakamura, Y., et al (1997b) Subjective experience and related symptoms in schizophrenia. Comprehensive Psychiatry, 38, 49-55.

Kirov, G., Kemp, R., Kirov, K., et al (1998) Religious faith after psychotic illness. Psychopathology, 31, 234-245.

Kjellin, L., Andersson, K., Candefjord, I. L., et al (1997) Ethical benefits and costs of coercion in short-term inpatient psychiatric care. Psychiatric Services, 48, 1567-1570.

Lam, D. \& Wong, G. (1997) Prodromes, coping strategies, insight and social functioning in bipolar affective disorders. Psychological Medicine, 27, 1091-1100.

Laws, K. R. (1999) A meta-analytic review of Wisconsin Card sort studies in schizophrenia: a general intellectual deficit in disguise. Cognitive Neuropsychiatry, 4, 1-35.

Lewis, A. (1934) The psychopathology of insight. British Journal of Medical Psychology, 14, 332-348.

Lysaker, P. H., Bell, M. D., Bryson, G., et al (1998a) Neurocognitive function and insight in schizophrenia: support for an association with impairments in executive function but not with impairments in global function. Acta Psychiatrica Scandinavica, 97, 297-301.

-, Bell, M. D., Bryson, G. J., et al (1998b) Insight and interpersonal function in schizophrenia. Journal of Nervous and Mental Disease, 186, 432-436.
MacPherson, R., Jerrom, B. \& Hughes, A. (1996) A controlled study of education about drug treatment in schizophrenia. British Journal of Psychiatry, 168, 709-717.

McEvoy, J. P. (1998) The relationship between insight in psychosis and compliance with medications. In Insight and Psychosis (eds X. F. Amador \& A. S. David), pp.289306. New York: Oxford University Press.

-, Apperson, L. J., Appelbaum, P. S., et al (1989a) Insight in schizophrenia: its relationship to acute psychopathology. Journal of Nervous and Mental Disease, 177, 43-47.

-, Appelbaum, P. S., Geller, J. L., et al (1989b) Why must some schizophrenic patients be involuntary committed? The role of insight. Comprehensive Psychiatry, 30, 13-17.

-, Schooler, N. J., Friedman, E., et al (1993) Use of psychopathology vignettes by patients with schizophrenia or schizoaffective disorder and by mental health professionals to judge patients' insight. American Journal of Psychiatry, 150, 1649-1653.

McNally, S. E. \& Goldberg, J. O. (1997) Natural cognitive coping strategies in schizophrenia. British Journal of Medical Psychology, 70, 159-167.

Markova, I. S. \& Berrios, G. E. (1992) The assessement of insight in clinical psychiatry: a new scale. Acta Psychiatrica Scandinavica, 86, 159-164.

Michalakeas, A., Skoutas, C., Charalambous, A., et al (1994) Insight in schizophrenia and mood disorders and its relation to psychopathology. Acta Psychiatrica Scandinavica, $90,46-49$

Nayani, T. H. \& David, A. S. (1996) The auditory hallucination - A phenomenological survey. Psychological Medicine, 26, 177-189.

Overall, J. \& Gorham, D. (1962) The Brief Psychiatric Rating Scale. Psychological Reports, 10, 799-812.

Peralta, V. \& Cuesta, M. J. (1998) Lack of insight in mood disorders. Journal of Affective Disorders, 49, 55-58.

Perkins, R. \& Moodley, P. (1993) The arrogance of insight? Psychiatric Bulletin, 17, 233-234.

Rossell, S. \& David, A. (1997) Improving performance in WCST: variations on the original procedure. Schizophrenia Research, 28, 63-76.

Sanz, M., Constable, G., Lopez-Ibor, I., et al (1998) A comparative study of insight scales and their relationship to psychopathological and clinical variables. Psychological Medicine, 28, 437-446.

Smith, C. M., Barzman, D. \& Pristach, C. A (1997) Effect of patient and family insight on compliance of schizophrenic patients. Journal of Clinical Psychopharmacology, 37, 147154.

Startup, M. (1996) Insight and cognitive deficits in schizophrenia: evidence for a curvilinear relationship. Psychological Medicine, 26, 1277-1281.

- (1997) Awareness of own and others' schizophrenic illness. Schizophrenia Research, 26, 203-211.

Sullwold, L. (1986) Frankfurt Complaints Questionnaire (3). In Schizophrenic Basic Disorders (in German) (eds L. Sullwold, G. Huber), pp. 145-159. Berlin: Springer.

Swanson, C. L., Freudenreich, O., McEvoy, J. P., et al (1995) Insight in schizophrenia and mania. Journal of Nervous and Mental Disease, 183, 752-755

Tan, T. (1993) Assessment of insight in Singapore. British Journal of Psychiatry, 162, 565-566.

Wahass, S. \& Kent, G. (1997) Coping with auditory hallucinations: A cross-cultural comparison between Western (British) and Non-Western (Saudi Arabian) patients. Journal of Nervous and Mental Disease, 185, 664668.

Wing, J. K., Cooper, J. E., Sartorius, N. (1974) The Description and Classification of Psychiatric Symptoms: An Instruction Manual for PSE and Catego System. London: Cambridge University Press.

World Health Organization (1973) Report of the International Pilot Study of Schizophrenia. Geneva: WHO.

Young, D. A, Zakzanis, K. K., Bailey, C., et al (1998) Further parameters of insight and neuropsychological deficit in schizophrenia and other chronic mental disease. Journal of Nervous and Mental Disease, 186, 44-50. 


\section{Multiple choice questions}

1. The following factors may be considered dimensions of insight:

a awareness of having a mental illness

b ability to recognise the pathological symptoms in others

c perceived need for treatment

d knowledge of presentations of mental disorder.

2. Regarding insight and psychopathology:

a recovery from psychopathology underlies the improvement of insight

b patients with neurotic disorders always have preserved insight

c depressive disorders may be associated with increase of insight

d subjective experiences of psychosis are identical to awareness of illness.

3. Patients may develop natural strategies to cope with the following:
a hallucinations
b prodromes of depression
c prodromes of mania
d 'basic symptoms' of schizophrenia.

4. Increase in adherence to treatment may result from:
a psychoeducation
b compliance therapy
c enforced compliance
d substance misuse.

5. The following are associated with improved insight into psychosis:
a psychoanalysis
b depressed mood
c above average intelligence
d denial.

MCQ answers

$\begin{array}{llllllllll}\text { T } & & \text { 2 } & & 3 & & 4 & & 5 & \\ \text { a } & \text { T } & \text { a } & \text { F } & \text { a } & \text { T } & \text { a } & \text { T } & \text { a } & \text { F } \\ \text { b } & \text { F } & \text { b } & \text { F } & \text { b } & \text { T } & \text { b } & \text { T } & \text { b } & \text { T } \\ \text { c } & \text { T } & \text { c } & \text { T } & \text { c } & \text { T } & \text { c } & \text { T } & \text { c } & \text { T } \\ \text { d } & \text { F } & \text { d } & \text { F } & \text { d } & \text { T } & \text { d } & \text { F } & \text { d } & \text { F }\end{array}$

